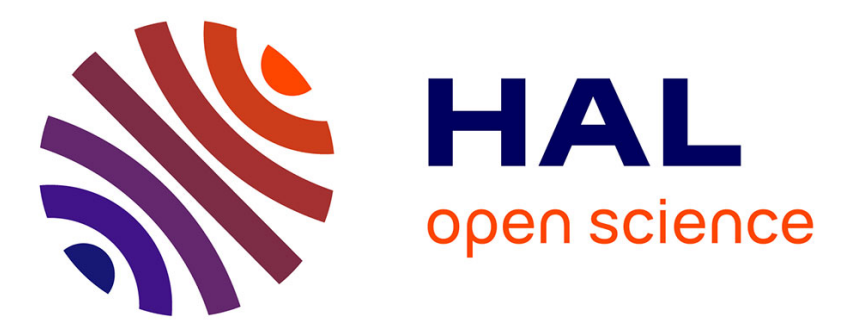

\title{
Electron-Phonon Scattering in 2D Silver Nanotriangles
}

Benoît Dacosta Fernandes, Aurélie Le Beulze, Fabien Moroté, Jean Oberlé,

Mona Tréguer-Delapierre, Julien Burgin, Pierre Langot

\section{To cite this version:}

Benoît Dacosta Fernandes, Aurélie Le Beulze, Fabien Moroté, Jean Oberlé, Mona Tréguer-Delapierre, et al.. Electron-Phonon Scattering in 2D Silver Nanotriangles. Journal of Physical Chemistry C, 2013, 117 (42), pp.22041-22045. 10.1021/jp406357p . hal-00879092

\section{HAL Id: hal-00879092 \\ https://hal.science/hal-00879092}

Submitted on 6 Mar 2018

HAL is a multi-disciplinary open access archive for the deposit and dissemination of scientific research documents, whether they are published or not. The documents may come from teaching and research institutions in France or abroad, or from public or private research centers.
L'archive ouverte pluridisciplinaire HAL, est destinée au dépôt et à la diffusion de documents scientifiques de niveau recherche, publiés ou non, émanant des établissements d'enseignement et de recherche français ou étrangers, des laboratoires publics ou privés.

\section{다)(1) $(5$}

Distributed under a Creative Commons Attribution - NonCommercial| 4.0 International 


\title{
Electron-Phonon Scattering in 2D Silver Nanotriangles
}

\author{
Benoît Dacosta Fernandes, ${ }^{\dagger}$ Aurélie Le Beulze, ${ }^{\ddagger}$ Fabien Moroté, ${ }^{\dagger}$ Jean Oberlé, ${ }^{\dagger}$ Mona Tréguer-Delapierre, ${ }^{\ddagger}$ \\ Julien Burgin, ${ }^{\dagger}$ and Pierre Langot* ${ }^{\dagger} \dagger$ \\ ${ }^{\dagger}$ Univ. Bordeaux, LOMA, UMR 5798, F 33400 Talence, France \\ ${ }^{\ddagger}$ CNRS, Univ. Bordeaux, ICMCB, UPR 9048, F 33600 Pessac, France
}

\begin{abstract}
Electron-phonon energy exchanges are investi gated in 2D silver nanotriangles of thickness ranging from 5 to $8 \mathrm{~nm}$ and lateral size ranging from 25 to $85 \mathrm{~nm}$, using time resolved femtosecond spectroscopy in the low perturbation regime. The measured electron-phonon decay time is smaller in $2 \mathrm{D}$ nano triangles than in bulk silver, and its value corresponds to the decay time measured in isolated nanospheres with a diameter equal to the thickness of the nanotriangles. These results show that the electron-phonon energy exchanges in $2 \mathrm{D}$ nanosystems are strongly accelerated by confinement and this acceleration is directly governed by the smallest dimension of the nano object.
\end{abstract}

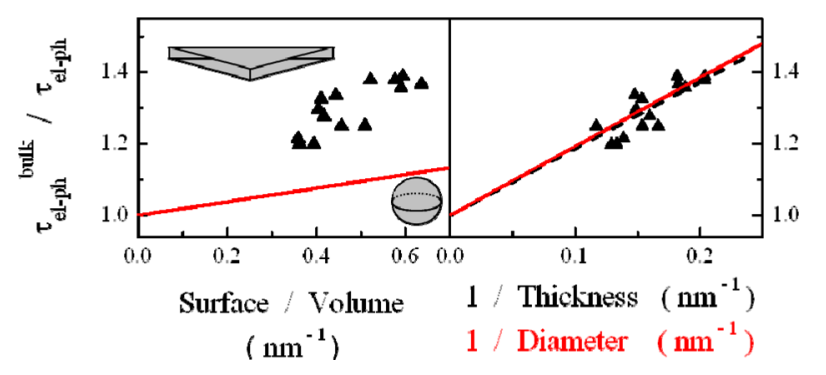

\section{INTRODUCTION}

The effect of confinement on the electron dynamics in nanometer sized metal particles has been intensively studied over the last few decades. ${ }^{1-4}$ The interactions of electrons between themselves and with their environment are strongly modified close to a surface or by confinement in systems of reduced dimensionality. Time resolved femtosecond spectros copy is a powerful technique for the investigation of the different electron scattering processes that occur in metals. The first studies investigated these properties in bulk noble metallic systems. $^{5-7}$ Later, this approach was extended to metal nanoparticles such as gold, silver, gallium, and tin and on bimetallic compounds. ${ }^{8-18}$ More recently, experiments on small clusters showed that quantum size effects could affect electron-phonon processes in spheres smaller $2-3 \mathrm{~nm}{ }^{19,20}$ However, one has to mention that contradictory results about the size effects have been reported on these studies. The discrepancies result from the pump intensity dependence of the measured dynamics which is mainly due to the temperature dependence of the electronic heat capacity. ${ }^{21}$ Very important information about confinement effects were obtained by Arbouet et al., particularly concerning the acceleration of the electron-lattice energy exchanges for spheres smaller than 10 nm. ${ }^{10}$ Femtosecond pump-probe experiments performed in the low perturbation regime have demonstrated a strong increase of the intrinsic electron-phonon interactions for noble metal nanoparticles smaller than $10 \mathrm{~nm}$ due to a confinement effect independent of the nanoparticle environ ment and the synthesis technique. ${ }^{10}$ However, the quantitative interpretation of electron-phonon coupling in metallic clusters remains challenging even for pure noble particles, and additional experimental and theoretical investigations are needed to fully determine the mechanisms involved in energy exchanges at the nanoscale in confined systems. Indeed, using simple arguments, one can consider that the dependence of the electron-phonon coupling constant is related to the surface to volume ratio. ${ }^{10,12,15,16,23,29}$ The extension of these pioneering experiments on size effects in nanospheres to other types of confined systems (1D or 2D) would be particularly interesting to investigate this argument. In this context, as the control of $2 \mathrm{D}$ metal nanosystems synthesis is becoming very efficient, ${ }^{22}$ we can now envisage addressing $2 \mathrm{D}$ geometries and try to go further in the understanding of electron-phonon scattering phenomena at the nanoscale in metals.

In the present work, the effect of surfaces on the electronphonon thermalization dynamics is studied in $2 \mathrm{D}$ silver nanotriangles of thickness ranging from 5 to $8 \mathrm{~nm}$ and length ranging from 25 to $85 \mathrm{~nm}$ using a high sensitivity femtosecond pump-probe setup. The results show that the characteristic electron-phonon (el-ph) coupling time $\tau_{\mathrm{el}-\mathrm{ph}}$ measured in 2D nanotriangles corresponds to the one obtained in silver nanospheres with a diameter equal to the nanotriangle thickness. Our study yields evidence that $\tau_{\mathrm{el}-\mathrm{ph}}$ is not simply the measured time in bulk metal $\left(\tau_{\mathrm{el}-\mathrm{ph}}\right.$ bulk $)$ modified by a quantity proportional to the surface to volume ratio as expected from recent studies. ${ }^{10,23,24}$

\section{EXPERIMENTAL METHODS}

The pump-probe femtosecond techniques allow us to monitor the energy loss of the photoexcited electron gas to the lattice vibrations. It has been shown that, for a probe photon energy well below the threshold of interband transitions, the signal 
amplitude is only weakly sensitive to the electron distribution and is almost proportional to the electron gas excess energy (defined as the difference between the electron gas energy at time $t_{\mathrm{D}}$ and the one before perturbation, $t_{\mathrm{D}}$ being the pumpprobe delay). ${ }^{25,26}$ In the low perturbation regime (electronic temperature rise $\Delta T_{\text {exc }}$ smaller than $200 \mathrm{~K}$ ), the electron gas excess energy is transferred to phonons according to a monoexponential decay with a characteristic time $\tau_{\text {el-ph }}$ in agreement with the two temperature model..$^{21,27}$ In these conditions, the time evolution of the measured signal $\Delta T / T\left(t_{\mathrm{D}}\right)$ directly reflects the energy losses of the electrons to their environment, permitting the precise analysis of electron-phonon interactions. At longer time scales, the measured signal reflects the acoustic vibrations of the nanosystems and the energy transfer to the environment. The periods of the measured vibrations are directly related to the size and geometry of the nanosystems. ${ }^{28-32}$

The Ag nanotriangles studied in this work have been synthesized using seed mediated growth because it represents a simple and versatile route for large scale production. As an advantage over conventional methods based on homogeneous nucleation, seed mediated growth allows one to disentangle growth from nucleation, making it easier to control both the size and shape of a product by simply varying concentration on the growth step. ${ }^{33,34}$ In a typical procedure, small silver spheres were first synthesized in an aqueous solution by reducing $\mathrm{AgNO}_{3}$ with $\mathrm{NaBH}_{4}$ in the presence of poly(styrenesulfonate) (PSS) and sodium citrate. The average diameter of these nanoparticles is $3.3 \pm 0.8 \mathrm{~nm}$. The absorption spectrum of the colloidal suspension exhibited a sharp band at $383 \mathrm{~nm}$, characteristic of dipole resonance of the small silver nano spheres. The full width at half maximum was found around 43 $\mathrm{nm}$, confirming the monodispersity of the produced particles. When a small amount of this dispersion was introduced in a growth medium containing $\mathrm{AgNO}_{3}$, L ascorbic acid (AA), PVP, and citrate at room temperature, the silver nanospheres were completely transformed into thin and flat nanostructures, yielding a transparent blue dispersion with an absorbance maximum in the near infrared. Figure 1d shows the normalized UV-visible spectrum of the resulting colloidal suspension of the Ag products. Four localized surface plasmon resonance peaks are observed, confirming the presence of thin anisotropic nanostructures once the seeded growth was initiated. The dominant extinction peak at $700 \mathrm{~nm}$ is the in plane dipole resonance mode, whereas the three weak bands near 332, 450, and $500 \mathrm{~nm}$ are the out of plane quadrupole mode, out of plane dipole mode, and in plane quadrupole mode, respec tively. ${ }^{35}$ These observations are consistent with the character istic dipole and quadrupole resonance peaks of $50 \mathrm{~nm}$ long triangular Ag nanostructures. ${ }^{36-38}$ Transmission electron microscopy (TEM) images of these nanostructures are shown in Figure 1. The prevailing shapes are triangular nanostructures $(85 \%)$ coexisting with hexagonal and circular nanostructures (15\%). Note that the triangular nanotriangles have a slight truncation at the corners (Figure 1b). As observed when they were stacked perpendicular to the substrate (Figure 1c), they were thin and flat in agreement with absorption spectroscopy measurements. Their thickness is found to be around $5 \mathrm{~nm}$, which is close to the dimension of the seeds. The size (length) of the anisotropic nanostructures could be reliably controlled by varying the amount of silver ions mixed with a specific quantity of seeds or by varying the quantity of seeds added to a solution containing a specific amount of $\mathrm{Ag}^{+}$. As expected, a

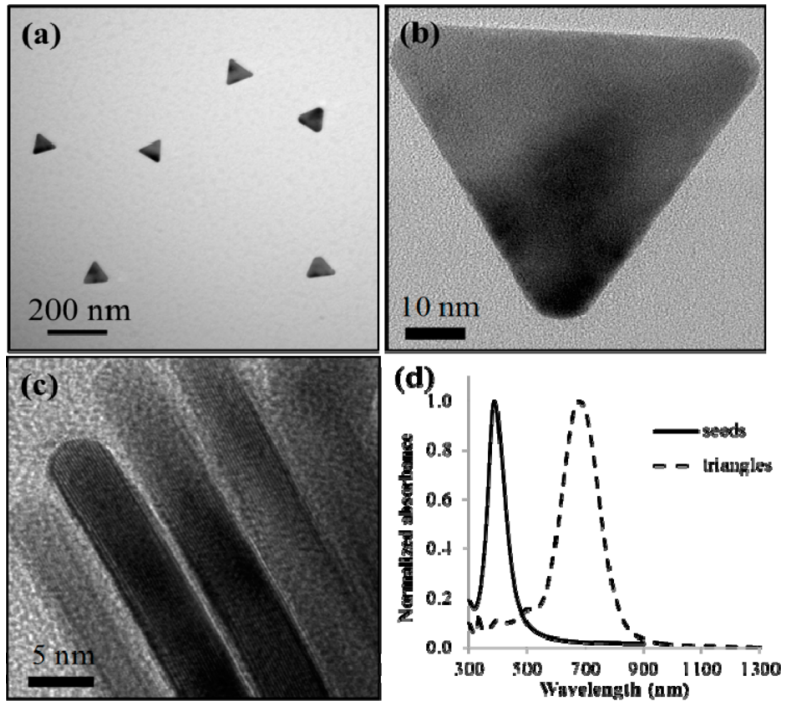

Figure 1. $(\mathrm{a}-\mathrm{c})$ TEM and high resolution TEM images of the Ag nanotriangles synthesized in a growth medium containing PVP and citrate. (d) Extinction spectrum of the Ag spherical seeds and $\mathrm{Ag}$ nanotriangles.

larger amount of ionic precursor or a lower amount of seeds leads to the formation of larger nanotriangles. Control of the thickness of the flat nanostructures is more difficult to achieve. One observes that replacing the citrate with carboxylate like compounds with lower $\mathrm{COO}^{-}$groups interferes with the kinetic growth of the anisotropic objects and allows the production of thicker nano objects. A carboxylate compound with only two $\mathrm{COO}^{-}$groups, such as succinate, permits the thickness to increase up to $8 \mathrm{~nm}$.

The experimental pump-probe spectroscopy setup is based on a commercial high repetition rate Ti:Sapphire femtosecond laser source $(150 \mathrm{fs}, 680-1080 \mathrm{~nm}, 80 \mathrm{MHz})$. The pulse train is split into two parts. The first part, the pump pulse, is used to create a non equilibrium electron distribution at either the fundamental or doubled frequency of the laser. The second part, the probe pulse, at the fundamental laser frequency, is used to monitor the induced sample transmission changes $\Delta T$ / $T$ for several delay times $t_{\mathrm{D}}$. The detection is performed with mechanical chopping of the pump beam at $1.5 \mathrm{kHz}$ and lock in differential detection of the transmitted probe beam intensity. The high stability and repetition rate of our setup with a noise level in the $10^{-7}$ range enable us to achieve high sensitivity measurements in the low perturbation regime with $\Delta T_{\mathrm{exc}}<200$ K .

\section{RESULTS AND DISCUSSION}

The experiments were performed in colloidal solutions of $2 \mathrm{D}$ silver nanotriangles with lateral sizes $L$ ranging from 25 to 85 $\mathrm{nm}$ and the thickness $H$ ranging between 5 and $8 \mathrm{~nm}$ in a $1 \mathrm{~mm}$ thick Hellma quartz cell. The temporal evolution of $\Delta T / T$ measured in $2 \mathrm{D}$ silver nanotriangles with a lateral size of $65 \mathrm{~nm}$ is shown in Figure 2a. After a sharp rise in the first hundreds of femtoseconds following energy injection, $\Delta T / T$ decreases and represents energy losses from the electron gas to the lattice. Assuming that both the electron gas and the lattice are thermalized (typically for $t_{\mathrm{D}}>800 \mathrm{fs}$ ), ${ }^{39}$ these energy exchanges can be described using the two temperature model. ${ }^{21,27}$ In the low perturbation regime, this model predicts a mono exponential decay of the electronic energy excess with the 

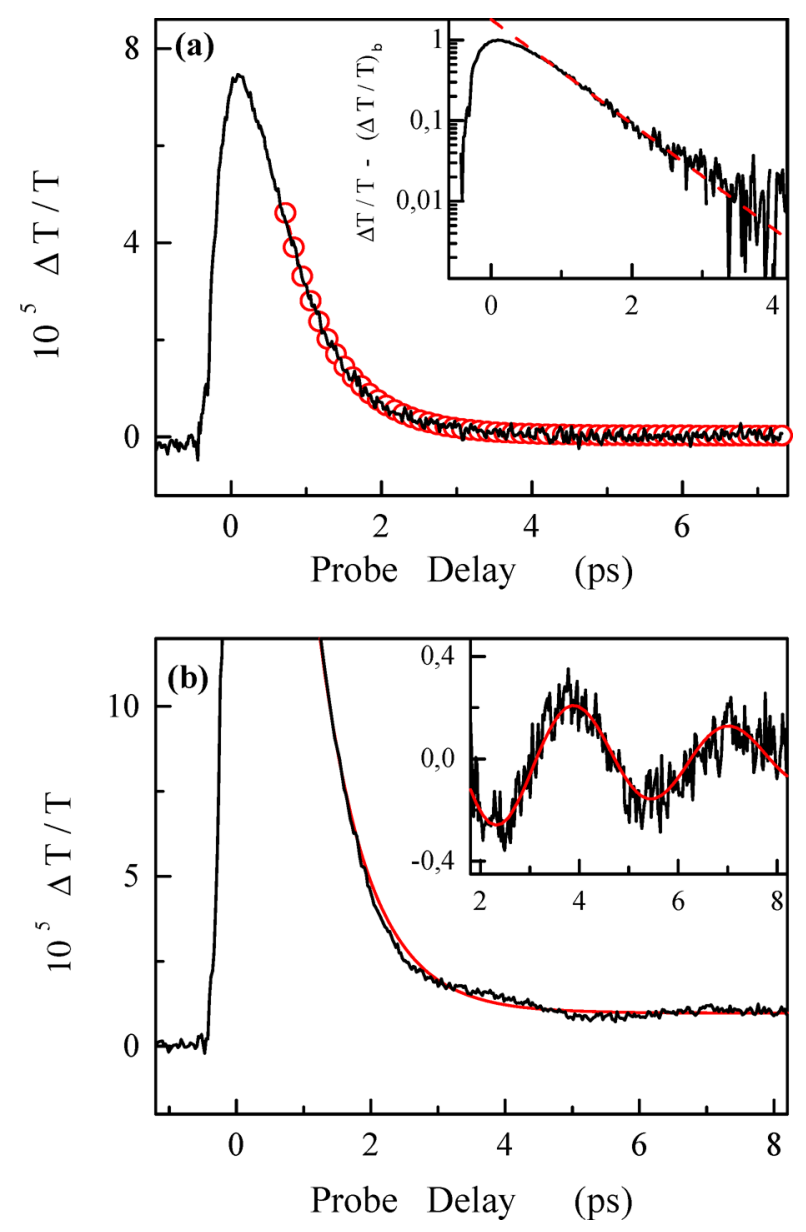

Figure 2. (a) Temporal evolution of the transmission change $\Delta T / T$ measured in $2 \mathrm{D} \mathrm{Ag}$ nanotriangles colloidal solution $\left(\lambda_{\text {pump }}=\lambda_{\text {probe }}=\right.$ $780 \mathrm{~nm}$ ) with average pumping power of $1 \mathrm{~mW}$. The lateral size of the nanotriangles is $65 \pm 13 \mathrm{~nm}$. The inset shows the same data on a logarithmic scale after subtraction of the long delay background and a fit using an exponential decay with a coupling time $\tau_{\text {el-ph }}=675$ fs (red, dashed line). (b) Transmission changes $\Delta T / T$ measured in the same sample with a pump power of $4 \mathrm{~mW}$. The red line is a fit by a monoexponential decay. Inset: The oscillating signal (black line) is the difference between the two previously mentioned curves, and the red line is the numerical fit of the oscillating part with one damped cosine function.

previously mentioned characteristic time $\tau_{\mathrm{el}-\mathrm{ph}}{ }^{10,40}$ The latter can thus be extracted by fitting the experimental data with a single decayed exponential function (Figure 2a). In $65 \mathrm{~nm}$ triangles, we obtained a characteristic time $\tau_{\mathrm{el}-\mathrm{ph}}=675 \pm 20 \mathrm{fs}$ for both excitation configurations $\left(\lambda_{\text {pump }}=780 \mathrm{~nm}\right.$ and $\lambda_{\text {pump }}=$ $390 \mathrm{~nm})$ and for an off resonance probe $\left(\lambda_{\text {probe }}=780 \mathrm{~nm}\right)$. Naturally, we checked that the time extracted from our measurements was independent of the pump power in order to ensure that we worked in the weak perturbation regime. ${ }^{10,21,29}$ Measurements performed in the different samples show similar behaviors with a decay time much shorter than the one determined for bulk silver $\left(\tau_{\mathrm{el}-\mathrm{ph}}\right.$ bulk $\left.=875 \mathrm{fs}\right)$, yielding evidence for a confinement induced acceleration of the electronphonon energy exchanges in 2D nanotriangles. Previous systematic investigations of the size effects on the electron internal thermalization and electron-lattice thermalization in spherical metal nanoparticles have pointed out an acceleration of these electronic processes with decreasing size. Though a full theoretical model has yet to be developed, this has been attributed to an enhancement of the electron-ion coupling close to the nanoparticle surface due to reduction of the screening of the Coulombic interactions. The importance of this effect increases with size reduction and become very efficient under $10 \mathrm{~nm}$ when surface effects dominate volume effects. As a first approximation, the electron-phonon energy exchange rate $\left(1 / \tau_{\mathrm{el}-\mathrm{ph}}\right)$ can be written as the sum of a volume and a surface contribution. The latter is responsible for the increase of the electron-lattice coupling with nanoparticle size reduction. If we normalize the energy exchange rate in the nanosystems over the rate in the bulk material, which contains only the term proportional to the volume, we can obtain the expression $\tau_{\mathrm{el}-\mathrm{ph}}^{\text {bulk }} / \tau_{\mathrm{el}-\mathrm{ph}}=1+\alpha(S / V)$. This is used to reproduce the experimental results obtained in nanospheres (Figure 3a). To interpret our results and compare them with previous studies, we first assumed that the relevant parameter was also the surface to volume ratio. For a nanotriangle,
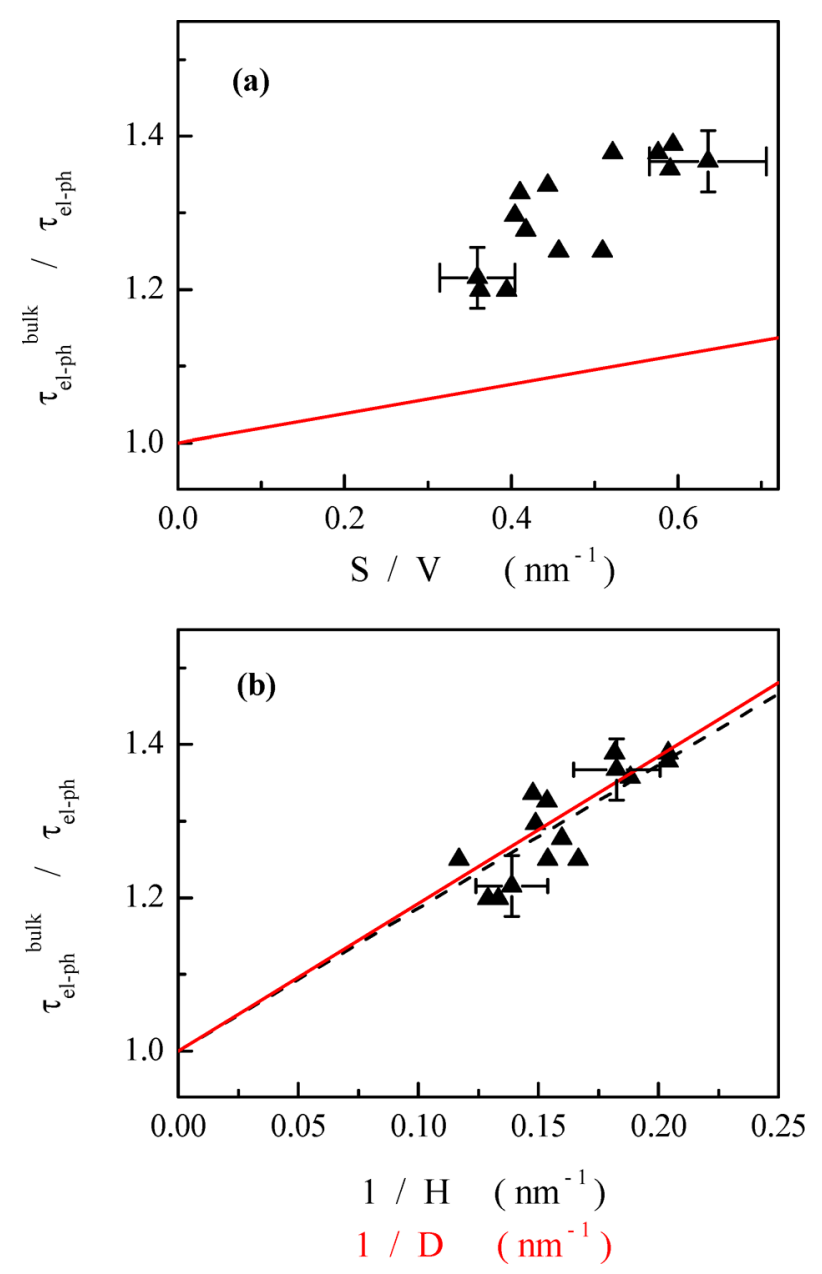

Figure 3. (a) Variation of the inverse electron-phonon time normalized to the bulk time $\left(\tau_{\mathrm{el}-\mathrm{ph}}\right.$ bulk $)$ as a function of surface to volume ratio measured in $2 \mathrm{D} \mathrm{Ag}$ nanotriangles $(\boldsymbol{\Delta})$. The red line mimics the evolution of $\left(\tau_{\mathrm{el}-\mathrm{ph}}\right.$ bulk $\left./ \tau_{\mathrm{el}-\mathrm{ph}}\right)$ measured in Ag nanospheres by Arbouet et al. ${ }^{10}$ (b) Evolution of $\left(\tau_{\mathrm{el}-\mathrm{ph}}\right.$ bulk $\left./ \tau_{\mathrm{el}-\mathrm{ph}}\right)$ as a function of the inverse of nanotriangle thickness $(\boldsymbol{\Lambda})$. The red line mimics the variation of $\left(\tau_{\mathrm{el}-\mathrm{ph}}\right.$ bulk $\left./ \tau_{\mathrm{el}-\mathrm{ph}}\right)$ of Ag nanospheres measured by Arbouet et al. ${ }^{10}$ as a function of the inverse of their diameter. The dashed line corresponds to a linear fit with $\tau_{\text {el-ph }}$ bulk $/ \tau_{\text {el-ph }}=1+\beta / H$, where $H$ is the thickness of the nanotriangles. 
$(S / V)_{\text {triangle }}=2 / H+4 \sqrt{ } 3 / L$, whereas for a nanosphere with a diameter $D,(S / V)_{\text {sphere }}=6 / D$. To estimate the surface to volume ratio of our nanotriangles, we needed to determine precisely both dimensions. The length $L$ is precisely measured with TEM microscopy, but the height $H$ is difficult to access with this technique (Figure 1a,b). Indeed, even if several pictures for some samples showed triangles lying on their side (Figure 1c), we could not get good statistics on this dimension. Meanwhile, as previously mentioned, our ultrafast pumpprobe technique is sensitive to acoustic vibrations, and these vibrations are directly related to the particles dimensions. ${ }^{28,32}$ Then, to determine the thickness of every sample of nanotriangles, we performed measurements in the high excitation regime to observe significant vibrational signals. This technique is relevant here because it measures simultaneously the vibrations of thousands of individual particles oscillating in phase and makes an ensemble average of their thickness. The relative transmission change displays small oscillations (which partially overlap with its short delay decays) that correspond to the thickness vibration mode (Figure 2b). This mode has already been observed and studied in nanoprisms and nanoplatelets and is here used to characterize precisely the size distribution. ${ }^{31,32}$ To convert the measured periods into thickness $H$, we used a computer program based on the resolution of the Navier-Stokes equation using finite element analysis (COMSOL). This approach allowed us to obtain the surface to volume ratio of every nanotriangle sample from the TEM measured size $L$ and the measured period related to the height $H$ (see Supporting Information). For $65 \mathrm{~nm}$ nanotriangles, we measured a period of 3.5 ps which corresponds to a height $H=6.4 \mathrm{~nm}$, in agreement with TEM measurements. For this sample, we find a ratio $(S / V)_{\text {triangle }}=0.42$, corresponding to spheres with a diameter $D=14.6 \mathrm{~nm}$. For these spheres, an electron-phonon time of 820 fs has been measured. ${ }^{10}$ We thus find that in terms of electron-phonon energy exchanges, these $2 \mathrm{D}$ nanotriangles do not correspond to spheres having the same surface to volume ratio. To confirm this result, we performed measure ments on several sets of samples over a range of thickness $H$ (from $\sim 4.9$ to $\sim 7.2 \mathrm{~nm}$ ) and length $L$ (from $\sim 25.5$ to $\sim 85$ $\mathrm{nm}$ ), corresponding to surface volume ratio between $0.35 \pm$ 0.04 and $0.64 \pm 0.07$. The results are presented in Figure 3 and show that the evolution of $\tau_{\mathrm{el}-\mathrm{ph}}$ bulk $/ \tau_{\mathrm{el}-\mathrm{ph}}$ as a function of the surface to volume ratio is not in agreement with the data obtained by Arbouet et al. for spherical Ag nanoparticles. ${ }^{10}$ For the studied range of surface to volume ratios, the average electron-phonon time in 2D nanotriangles varies between 630 and $720 \mathrm{fs}$, whereas for nanospheres with the same surface to volume ratio it varies between 780 and 830 fs (Figure 3a). The important discrepancy of these results shows that surface to volume ratio is not a universally relevant parameter. However, the evolutions of $\tau_{\mathrm{el}-\mathrm{ph}}$ bulk $/ \tau_{\mathrm{el}-\mathrm{ph}}$ as a function of $1 / H$ for the nanotriangles and as a function of $1 / D$ for the nanospheres plotted in Figure $3 \mathrm{~b}$ are in excellent agreement. This shows that the smallest dimension of the nano object is the relevant parameter here. For silver nanotriangles with a large lateral size to thickness ratio (i.e., a small aspect ratio and $H<10 \mathrm{~nm}$ ), the characteristic decay time $\tau_{\mathrm{el}-\mathrm{ph}}$ corresponds to the one obtained in silver nanospheres with a diameter comparable to the thickness of the nanotriangles. This result is particularly interesting and shows that in these $2 \mathrm{D}$ nanosystems the confinement along a single dimension plays a very strong role in electron-phonon energy exchanges, i.e., it is not the surface to volume ratio which in important but rather the inverse of the smallest dimension. We can summarize that a one dimensional confinement $(<10 \mathrm{~nm})$ produces the same acceleration of electron-phonon energy exchanges as a three dimensional confinement. This result brings new insights in the under standing of electron-phonon scattering in nanometals and particularly will allow progress in the modeling of the role of surface effects on electron-lattice energy exchanges in confined systems.

\section{CONCLUSION}

In summary, we have performed an investigation of the electron-lattice energy exchanges in $2 \mathrm{D}$ silver nanotriangles using a time resolved femtosecond technique in the low perturbation regime. The confinement in systems of reduced dimensionality governs the acceleration of the electronphonon energy exchanges. In 2D nanosystems, the increase of the intrinsic electron-phonon interactions is due to confinement and is governed by the smallest dimension of the nano object (when it becomes smaller than $10 \mathrm{~nm}$ ) and not by the surface to volume ratio as proposed in earlier studies. The extension of these initial measurements in $2 \mathrm{D}$ systems to other noble metals such as gold and copper will bring new insights concerning this interesting behavior. To go further, the study of $1 \mathrm{D}$ systems such as nanorods or nanowires and the comparison with results obtained using other experimental techniques such as time resolved photoemission would be particularly interesting to fully understand this behavior.

\section{AUTHOR INFORMATION}

\section{Corresponding Author}

*E mail: pierre.langot@u bordeaux1.fr.

\section{Notes}

The authors declare no competing financial interest.

\section{ACKNOWLEDGMENTS}

The authors are grateful for funding support from the French Conseil Régional d'Aquitaine (Project Nano Trans 20111101010) and CNRS. The authors thank the Nano Spectro Imagery facility (NSI) at LOMA.

\section{REFERENCES}

(1) Halperin, W. P. Quantum Size Effects in Metal Particles. Rev. Mod. Phys. 1986, 58, 533-606.

(2) Link, S.; El Sayed, M. A. Spectral Properties and Relaxation Dynamics of Surface Plasmon Electronic Oscillations in Gold and Silver Nanodots and Nanorods. J. Phys. Chem. B 1999, 103, 84108426.

(3) Voisin, C.; Del Fatti, N .; Christofilos, D.; Vallée, F. Ultrafast Electron Dynamics and Optical Nonlinearities in Metal Nanoparticles. J. Phys. Chem. B 2001, 105, 2264-2280.

(4) Bigot, J. Y.; Halté, V.; Merle, J. C.; Daunois, A. Electron Dynamics in Metallic Nanoparticles. Chem. Phys. 2000, 251, 181-203.

(5) Schönlein, R. W.; lin, W. Z.; Fujimoto, J. G.; Eesley, G. L. Femtosecond Studies of Nonequilibrium Electronic Processes in Metals. Phys. Rev. Lett. 1987, 58, 1680-1683. 
(6) Sun, C. K.; Vallée, F.; Acioli, L. H.; Ippen, E. P.; Fujimoto, J. G. Femtosecond Tunable Measurement of Electron Thermalization in Gold. Phys. Rev. B 1994, 50, 15337-15348.

(7) Del Fatti, N.; Bouffanais, R.; Vallée, F.; Flytzanis, C. Nonequilibrium Electron Interactions in Metal Films. Phys. Rev. Lett. 1998, 81, 922-925.

(8) Hodak, J. H.; Henglein, A.; Hartland, G. V. Electron Phonon Coupling Dynamics in Very Small (between 2 and $8 \mathrm{~nm}$ diameter) Au Nanoparticles. J. Chem. Phys. 2000, 112, 5942-5947.

(9) Halté, V.; Bigot, J. Y.; Palpant, B.; Broyer, M.; Prével, B.; Pèrez, P. Size Dependence of the Energy Relaxation in Silver Nanoparticles Embedded in Dielectric Matrices. Appl. Phys. Lett. 1999, 75, 37993801.

(10) Arbouet, A.; Voisin, C.; Christofilos, D.; Langot, P.; Del Fatti, N.; Vallée, F.; et al. Electron Phonon Scattering in Metal Clusters. Phys. Rev. Lett. 2003, 90, 177401-177404.

(11) Jain, P. K.; Qian, W.; El Sayed, M. A. Ultrafast Electron Relaxation Dynamics in Coupled Metal Nanoparticles in Aggregates. J. Phys. Chem. B 2006, 110, 136-142.

(12) Nisoli, M.; Stagira, S.; De Silvestri, S.; Stella, A.; Togini, P.; Cheyssac, P.; Kofman, R. Ultrafast Electronic Dynamics in Solid and Liquid Gallium Nanoparticles. Phys. Rev. Lett. 1997, 78, 3575-3578.

(13) Permer, M.; Bost, P.; Lemmer, U.; Plessen, G. V.; Feldmann, J.; Becker, U.; Menning, M.; Schimdt, M.; Schimdt, H. Optically Induced Damping of the Surface Plasmon Resonance in Gold Colloids. Phys. Rev. Lett. 1997, 78, 2192-2195.

(14) Broyer, M.; Cottencin, E.; Lermé, J.; Pellerin, M.; Del Fatti, N.; Vallée, F.; Burgin, J.; Guillon, C.; Langot, P. Optical Properties and Relaxation Processes at Femtosecond Scale of Bimetallic Clusters. Faraday Discuss. 2008, 138, 137-145.

(15) Hodak, J. H.; Henglein, A.; Hartland, G. V. Tuning the Spectral and Temporal Response in PtAu Core-Shell Nanoparticles. J. Chem. Phys. 2001, 114, 2760-2765.

(16) Huang, W.; Qian, W.; El Sayed, M. A.; Ding, Y.; Wang, Z. L. Effect of the Lattice Crystallinity on the Electron-Phonon Relaxation Rates in Gold Nanoparticles. J. Phys. Chem. C 2007, 111, 1075110757.

(17) Tang, Y.; Ouyang, M. Tailoring Properties and Functionalities of Metal Nanoparticles Through Crystallinity Engineering. Nat. Mater. 2007, 6, 754-759.

(18) Knappenberger, K. L.; Schwartzberg, A. M.; Dowgiallo, A. M.; Lowman, C. A. Electronic Relaxation Dynamics in Isolated and Aggregated Hollow Gold Nanospheres. J. Am. Chem. Soc. 2009, 131, 13892-13893.

(19) Varnavski, O.; Ramakrishna, G.; Kim, J.; Lee, D.; Goodson, T. Critical Size for the Observation of Quantum Confinement in Optically Excited Gold Clusters. J. Am. Chem. Soc. 2010, 132, 16-17.

(20) Varnavski, O.; Ramakrishna, G.; Kim, J.; Lee, D.; Goodson, T. Optically Excited Acoustic Vibrations in Quantum Sized Monolayer Protected Gold Clusters. ACS Nano 2010, 4, 3406-3412.

(21) Del Fatti, N.; Arbouet, A.; Vallée, F. Femtosecond Optical Investigation of Electron-Lattice Interactions in an Ensemble and a Single Metal Nanoparticle. Appl. Phys. B: Lasers Opt. 2006, 84, 175181.

(22) Sau, T. K.; Rogach, A. L. Nonspherical Noble Metal Nanoparticles: Colloid Chemical Synthesis and Morphology Control. Adv. Mater. 2010, 22, 1781-1804.

(23) Darugar, Q.; Qian, W.; El Sayed, M. A.; Pileni, M. P. Size Dependent Ultrafast Electronic Energy Relaxation and Enhanced Fluorescence of Copper Nanoparticles. J. Phys. Chem. B. 2006, 110, $143-149$.

(24) Burgin, J.; Langot, P.; Arbouet, A.; Margueritat, J.; Mlayah, A.; Vallée, F.; Gonzalo, J.; Afonso, C. N.; Rossell, M.; Van Tendeloo, G. Acoustic Vibration Modes and Electron-Lattice Coupling in Self Assembled Silver Nanocolumns. Nano Lett. 2008, 8, 1296-1302.

(25) Del Fatti, N.; Voisin, C.; Achermann, M.; Tzortzakis, S.; Christofilos, D.; Vallée, F. Nonequilibrium Electron Dynamics in Noble Metals. Phys. Rev. B 2000, 61, 16956-16966.
(26) Groeneveld, R.; Sprik, R.; Lagendijk, A. Femtosecond Spectroscopy of Electron Electron and Electron Phonon Energy Relaxation in Ag and Au. Phys. Rev. B 1995, 51, 11433-11445.

(27) Kaganov, M. I.; Lifshitz, I. M.; Tanatarov, L. V. Relaxation between Electrons and the Crystalline Lattice. Zh. Eksp. Teor. Fiz. 1956, 31, 232; Soviet Phys. JETP 1957, 4, 173-178.

(28) Bonacina, L.; Callegari, A.; Bonati, C.; Van Mourik, F.; Chergui, M. Time Resolved Photodynamics of Triangular Shaped Silver Nanoplates. Nano Lett. 2006, 6, 7-10.

(29) Hartland, G. V. Optical Studies of Dynamics in Noble Metal Nanostructures. Chem. Rev. 2011, 111, 3858-3887.

(30) Tchebotareva, A. L.; Ruijgrok, P. V.; Zijlstra, P.; Orrit, M. Probing the Acoustic Vibrations of Single Metal Nanoparticles by Ultrashort Laser Pulses. Laser Photonics Rev. 2010, 4, 581-597.

(31) Burgin, J.; Langot, P.; Del Fatti, N.; Vallée, F.; Huang, W.; El Sayed, M. A. Time Resolved Investigation of the Acoustic Vibration of a Single Gold Nanoprism Pair. J. Phys. Chem. C. 2008, 112, 1123111235 .

(32) Fedou, J.; Viarbitskaya, S.; Marty, R.; Sharma, J.; Paillard, V.; Dujardin, E.; Arbouet, A. From Patterned Optical Near Fields to High Symmetry Acoustic Vibrations in Gold Crystalline Platelets. Phys. Chem. Chem. Phys. 2013, 15, 4205-4213.

(33) Millstone, J. E.; Hurst, S. J.; Métraux, G. S.; Cutler, J. I.; Mirkin, C. A. Colloidal Gold and Silver Triangular Nanoprisms. Small 2009, 5, 646-664.

(34) Pastoriza Santos, I.; Alvarez Puebla, R. A.; Liz Marzan, L. M. Synthetic Routes and Plasmonic Properties of Noble Metal Nano plates. Eur. J. Inorg. Chem. 2010, 2010, 4288-4297.

(35) Sherry, L. J.; Jin, R.; Mirkin, C. A.; Schatz, G. C.; Van Duyne, R. P. Localized Surface Plasmon Resonance Spectroscopy of Single Silver Triangular Nanoprisms. Nano Lett. 2006, 6, 2060-2065.

(36) Jin, R.; Cao, Y.; Mirkin, C. A.; Kelly, K. L.; Schatz, G. C.; Zheng, J. G. Photoinduced Conversion of Silver Nanospheres to Nanoprisms. Science 2001, 294, 1901-1903.

(37) Berriman, R. W.; Herz, R. H. Twinning and the Tabular Growth of Silver Bromide Crystals. Nature 1957, 180, 293-294.

(38) Sun, Y.; Mayers, B.; Xia, Y. Transformation of Silver Nanospheres into Nanobelts and Triangular Nanoplates through a Thermal Process. Nano Lett. 2003, 3, 675-679.

(39) Guillon, C.; Langot, P.; Del Fatti, N.; Vallée, F. Nonequilibrium Electron Energy Loss Kinetics in Metal Clusters. New J. of Phys. 2003, $5,13.1-13.9$

(40) Lermé, J.; Celep, G.; Broyer, M.; Cottancin, E.; Pellarin, M.; Arbouet, A.; Christofilos, D.; Guillon, C.; Langot, P.; Del Fatti, N.; Vallée, F. Effects of Confinement on the Electron and Lattice Dynamics in Metal Nanoparticles. Eur. Phys. J. D 2005, 34, 199-204. 